\title{
Paradiplomacia como Política Externa e Política Pública: modelo de análise aplicado ao caso da cidade do Rio de Janeiro
}

\author{
Paradiplomacy as Foreign Policy and Public Policy: \\ an analysis model applied to the case of the city of \\ Rio de Janeiro
}

DOI: $10.21530 /$ ci.v13n2.2018.790

Leonardo Mercher ${ }^{1}$

Alexsandro Eugenio Pereira ${ }^{2}$

\section{Resumo}

O presente artigo argumenta que estudos de paradiplomacia devem se aproximar dos estudos de política externa quando investigada como política pública. Essa seria uma estratégia para análise das práticas e estratégias das cidades nas relações internacionais. Os rendimentos analíticos dessa estratégia podem ser observados por meio de um modelo dimensional de análise de paradiplomacia como política externa e política pública. Esse modelo é proposto neste artigo e aplicado ao estudo empírico da cidade do Rio de Janeiro, no período de 1993 até 2016. O modelo sugere cinco dimensões explicativas nas análises da paradiplomacia: gestão política; mercado; institucional; internacional; e epistêmica. A aplicação desse modelo à cidade do Rio de Janeiro permitiu identificar as dimensões mais relevantes no caso específico da cidade - as dimensões política e de mercado - , enquanto que a variável partidária, defendida por outros pesquisadores do campo como de alta capacidade explicativa, pouco interferiu nos resultados observados durante a pesquisa.

Palavras-chave: Paradiplomacia; Política Externa; Política Pública; Rio de Janeiro.

\footnotetext{
1 Doutor em Ciência Política pela Universidade Federal do Paraná. Professor de Relações Internacionais no Centro Universitário Internacional (UNINTER), Curitiba, Paraná, Brasil.

2 Doutor em Ciência Política pela Universidade de São Paulo. Professor Associado dos Programas de Pós-Graduação em Ciência Política e em Políticas Públicasda Universidade Federal do Paraná, Curitiba, Paraná, Brasil.

Artigo submetido em 20/04/2018 e aprovado em 26/07/2018.
} 


\begin{abstract}
This paper argues that paradiplomacy studies should approach foreign policy studies when investigated as public policy. This would be a strategy to analyze the practices and strategies of cities in international relations. The analytical yields of this strategy can be observed through a dimensional model of paradiplomacy analysis such as foreign policy and public policy. This model is proposed in this article and applied to the empirical study of Rio de Janeiro, from 1993 to 2016. The model suggests five explanatory dimensions in paradiplomacy analyzes: political management; market; institutional; international; and epistemic. The application of this model to the City of Rio de Janeiro allowed us to identify the most relevant dimensions in this specific case - political and market dimensions - while the political party variable, defended by other researchers in the field as high explanatory capacity, had less interference in the results observed in this research.
\end{abstract}

Keywords: Paradiplomacy; Foreign Policy; Public Policy; Rio de Janeiro.

\title{
Introdução
}

Os estudos de paradiplomacia se deparam com limitações decorrentes do uso e da escolha de métodos de análise. Questionamentos de pesquisadores em diversos encontros de relações internacionais nos últimos anos, como os da Associação Brasileira de Relações Internacionais (ABRI), são recorrentes. As razões pelas quais estudos sobre cidades não avançarem para além de análises descritivas de suas práticas nos levaram a investigar o desenvolvimento da interdisciplinaridade entre os pesquisadores de relações internacionais e, com isso, defender a utilização da interdisciplinaridade de recursos e métodos de outras ciências, como da própria ciência política. Para que haja a ampliação das análises descritivas dos agentes seria então necessário problematizar e identificar variáveis sobre as estratégias, elaboração de agendas, meios de ação e cooperação e outras práticas e decisões das cidades.

Ao compreender a paradiplomacia como política externa e, consequentemente, como política pública, abre-se um leque de possibilidades analíticas e metodológicas para compreender mais criticamente a ação das cidades. Tanto os estudos de análise de política externa (APE) como os de análise de políticas públicas (APP) auxiliam a delimitar o tema e encontrar novos métodos para compreender as dinâmicas externas e internas à paradiplomacia. Essa percepção, no entanto, não esteve no horizonte dos primeiros estudos sobre a paradiplomacia, desenvolvidos 
por Panayotis Soldatos (1990) e Ivo Duchacek (1990)33. James Rosenau (1990), Soltados (1990) e Duchacek (1990) apontavam mais para as razões pelas quais um governo subnacional poderia ser visto como agente internacional do que para seus processos internos.

Durante os anos de 1990, a maior parte das publicações, como a de Brian Hocking (1993), estava mais preocupada com a contextualização das cidades nas relações internacionais do que em trazer métodos de investigação da formulação e execução da paradiplomacia. Mas por que olhar para dimensões internas à paradiplomacia? Muitas perspectivas teóricas das relações internacionais, como as institucionais, as funcionalistas ou as construtivistas, defendem que o agente (Estado) não é apenas resultado de estruturas e do cenário internacional, mas possui dinâmicas internas tão determinantes quanto as demandas externas para a elaboração de suas agendas (ROSENAU, 1967; WENDT, 1992; MILNER, 1997; FEARON, 1998; SALOMÓN, 2016).

Dessa forma, no presente artigo, busca-se contribuir com as análises mais amplas da paradiplomacia, ilustradas pelo caso da cidade do Rio de Janeiro como um agente governamental que, assim como os Estados nacionais, também experimenta fluxos e fenômenos internos que interferem em sua ação externa. A escolha do Rio justifica-se por duas razões principais: 1) por ser a cidade brasileira pioneira na criação de uma estrutura institucional específica para a atuação externa: "Desde 1986 - quando pela primeira vez no país um ente subnacional municipal cria uma estrutura de RI” (RIBEIRO, 2009, p. 96); e 2) pela existência de um conjunto de análises sobre a paradiplomacia, o planejamento e a relação da cidade com os grandes acontecimentos internacionais (Planos Estratégicos, ECO92, Rio +20, Jogos Olímpicos etc.), nos quais o município do Rio se viu envolvido (VAINER, 2001; SÁNCHEZ, 2001; AGUIAR, MENDES; SPADALE, 2013).

As investigações sobre a paradiplomacia utilizam determinadas variáveis explicativas como as partidárias, as institucionais e as de mercado (SALOMÓN; NUNES, 2007; LAISNER, 2007; RIBEIRO, 2009; ONUKI; OLIVEIRA, 2013). Essas investigações possibilitaram a construção e aplicação do modelo de análise de paradiplomacia (APD) sobre o Rio, proposto neste artigo. Esse modelo reúne diversas variáveis (e métodos de análise) em cinco dimensões que permitem examinar desde os gestores políticos (prefeitos e secretários), passando pelos

3 O conceito paradiplomacia foi formulado por Panayotis Soldatos (1990) e Ivo Duchacek (1990) e significa inicialmente diplomacia paralela ao governo central. Atualmente a compreensão do conceito é amplo e utilizado para se referir à ação externa de uma cidade ou outro governo local e subnacional nas relações internacionais. 
agentes do mercado, internacionais e epistêmicos. Para cada variável é preciso aplicar um método de coleta de dados. Com isso, o presente artigo traz resultados de diversas análises menores que formarão o todo, como as prosopografias de gestores políticos, de conselheiros, bem como do conteúdo de grupos epistêmicos (think tanks) ativos junto à cidade do Rio.

O presente artigo, além desta introdução, conta com duas seções e as considerações finais. O intuito da próxima seção é sustentar a necessidade de articulação entre os níveis externos e internos, que possibilita investigar os agentes, a formulação, a prática e as interferências dos ambientes externos e internos na paradiplomacia de uma cidade. Na seção posterior, o artigo apresenta o modelo de análise e o aplica à cidade do Rio de Janeiro no período de 1993 a 2016. Por fim, o artigo se encerra com as considerações finais, que destacam os possíveis rendimentos analíticos decorrentes da abordagem da paradiplomacia como política pública.

\section{Análise de Paradiplomacia como Análises de Política Externa e de Política Pública}

Quando se analisa paradiplomacia nas relações internacionais corre-se o risco de ignorar os processos internos das cidades e observar apenas suas ações no âmbito internacional. Por vezes, os estudos de redes de cidades, cooperação descentralizada ou a criação de políticas públicas advindas de regimes e acordos internacionais se tornam demasiadamente descritivos. Em um primeiro momento, é importante descrever os fatos e acontecimentos. Porém, diante do atual cenário acadêmico, é preciso avançar nas pesquisas críticas e na identificação de variáveis explicativas e intervenientes às ações paradiplomáticas.

Identificar padrões, exceções e variáveis explicativas do tipo de prática observada nas cidades possibilita a replicabilidade de métodos e análises científicos. Evidentemente que os casos apresentam particularidades e exigese investigação profunda em cada um deles. Mas é exatamente por isso que é necessário compartilhar modelos e técnicas que facilitem a comunicação inicial, como já ocorre na Análise de Política Externa (APE) e na Análise de Política Pública (APP).

A APE atualmente representa uma concepção metodológica e teórica de estudos de agendas estatais e política externa que leva em consideração os níveis 
domésticos e as interações entre agentes e instituições no âmbito nacional e internacional. É importante mencionar que foi durante os anos de 1960 e 1970 que alguns estudos sobre política externa tentaram compreender a ação externa dos Estados por meio de seus gestores - presidentes, ministros e instituições, como os de James Rosenau (1967) e Graham Allison (1969).

Nos anos de 1980, a ênfase na análise da política internacional predominou nas pesquisas de relações internacionais por causa, em parte, da influência do neorrealismo estrutural de Kenneth Waltz (1979). Porém, nos anos de 1990, algumas publicações buscaram retomar a abordagem de variáveis domésticas na explicação do comportamento dos Estados, como os estudos de Helen Milner e Robert Keohane (1996), que colocaram os interesses, as instituições e as informações no campo da política doméstica como variáveis explicativas à compreensão das ações do Estado nas relações internacionais. Nesse sentido, eles seguiram uma perspectiva estabelecida nos anos de 1950, quando surgiu a subdisciplina de "Análise de Política Externa" e, a partir desse momento, as variáveis domésticas tornaram-se importantes e entraram nas agendas de pesquisa de diferentes autores responsáveis pelo desenvolvimento dessa subdisciplina.

Partindo das percepções de interdependência complexa e dos múltiplos canais que ligam um Estado ao cenário internacional, Milner e Keohane (1996) identificaram mecanismos internos ao Estado, especialmente no âmbito da informação e das concepções sobre as ações políticas, que levariam às posições adotadas pela política externa. Seus estudos possibilitaram analisar a política interna e o papel dos agentes sociais internos ao Estado. Posteriormente, Valerie Hudson e Christopher Vore (1996) partiram do princípio segundo o qual os estudos sobre o comportamento dos Estados deveriam se iniciar pela compreensão de que o Estado seria composto por sociedades, instituições e dinâmicas que se cruzariam em diversos níveis de análise. Para tanto, os autores problematizaram cinco variáveis a serem analisadas: características individuais; percepções; sociedade e cultura; a política; e o sistema internacional.

Tanto nas análises no nível sistêmico da política internacional, como nas análises propriamente de APE, existiu o esforço de repensar o Estado em sua compreensão mais tradicional - de agente racional e coeso, tanto em suas dinâmicas internas como externas. Em James Fearon (1998) os estudos de APE deveriam identificar os grupos de interesses domésticos e a burocracia da política em questão (FEARON, 1998, p. 300) que permitissem traçar a construção de agendas e processos decisórios e estratégicos dos governos. Já Brian White (1999), 
por sua vez, aplica esses conceitos de agentes e fluxos internos/externos da APE para a União Europa (UE) como região. Brian White (1999) demonstrou que a agenda política da UE é consequência das relações de interesses e expectativas de seus Estados membros, sociedades e instituições. Com isso, White (1999) possibilita compreender que a política externa não está atrelada apenas ao Estado, mas a outras esferas de governos (instituições) e sociedade.

No Brasil, Mónica Salomón e Carmem Nunes (2007) propuseram esse olhar sobre os diversos níveis subnacionais. O olhar para as ideologias partidárias, instituições e outras variáveis domésticas foi aplicado no estudo paradiplomático da cidade de Porto Alegre e do estado do Rio Grande do Sul. Mais tarde, Mónica Salomón e Letícia Pinheiro (2013) problematizaram a APE, defendendo uma análise de variáveis externas e internas para se compreender as escolhas e ações estatais. Outros autores brasileiros aplicaram, também, os modelos de APE que levam em consideração esses multiníveis e variáveis internas e externas à paradiplomacia (LAISNER, 2007; ONUKI; OLIVEIRA, 2013), tendo contribuído para a compreensão da paradiplomacia como política externa.

No caso das cidades brasileiras, muito se avançou no processo de descentralização, sendo Brasil e Argentina os Estados com maior flexibilidade à ação de seus governos subnacionais no âmbito internacional (CGLU, 2016). Como atual cenário, diversas cidades e estados brasileiros hoje possuem secretarias ou coordenadorias de relações internacionais, bem como participam de diversas redes e organizações de cidades ao redor do mundo. Mas, como os casos empíricos apresentam particularidades, ao aplicar a APE nos estudos de paradiplomacia é preciso compreendê-la como política externa que, em sua essência, é, também, política pública. É por isso que nos estudos de Análise de Paradiplomacia (APD) por meio da APE é preciso recuperar algumas orientações do campo de Análise de Políticas Públicas (APP).

O agir externo das cidades pode ser compreendido como política pública levando em consideração as definições sugeridas por autores como Enrique Saraiva (2007) e Celina Maria de Souza (2006). A política pública, de modo geral, é compreendida como uma estratégia de elaboração e execução de ações que atendam aos interesses dos gestores da política e de quem eles deveriam representar (a população). Seguindo as indicações dos pesquisadores da APP, como Enrique Saraiva (2007) e Celina Maria Souza (2006), diversas são as frentes que buscam explicar o campo de políticas públicas: 
Das diversas definições e modelos sobre políticas públicas, podemos extrair e sintetizar seus elementos principais: I) A política pública permite distinguir entre o que o governo pretende fazer e o que, de fato, faz; II) A política pública envolve vários atores e níveis de decisão, embora seja materializada através dos governos, e não necessariamente se restringe a participantes formais, já que os informais são também importantes; III) A política pública é abrangente e não se limita a leis e regras; IV) A política pública é uma ação intencional, com objetivos a serem alcançados; V) A política pública, embora tenha impactos no curto prazo, é uma política de longo prazo; VI) A política pública envolve processos subsequentes após sua decisão e proposição, ou seja, implica também implementação, execução e avaliação. (SOUZA, 2006, p. 36-37)

Por isso, a APP leva em consideração variáveis como: partidos no poder; dinâmicas econômicas e institucionais; o papel da sociedade civil e dos interesses privados; e concepções de ação, como modelos de políticas públicas e boas práticas; dentre outras. Além disso, é importante identificar a natureza do agente investigado.

Como já é conhecida, a natureza jurídica e política das cidades se diferencia tradicionalmente dos Estados nacionais (ROSENAU, 1990). Sendo assim, quando métodos e modelos de análise são retirados da análise de políticas públicas, é necessário primeiramente traçar a natureza do agente. É necessário combinar, na análise empírica, elementos como: a natureza do agente (cidade, região, estado etc.); os cenários domésticos (desafios e oportunidades) e internacional (desafios e oportunidades); e as capacidades, desafios e demandas do agente. Com essa combinação de elementos, será possível desenvolver observações mais apuradas a respeito da ação externa das cidades.

No caso da APD, é preciso compreender a paradiplomacia como fruto da soma de três camadas tradicionais da APE (cenário internacional; governo; e cenário doméstico) combinadas com a natureza das cidades como agentes nas relações internacionais (Figura 1): 
Figura 1. Paradiplomacia como interseção de múltiplas camadas de análises

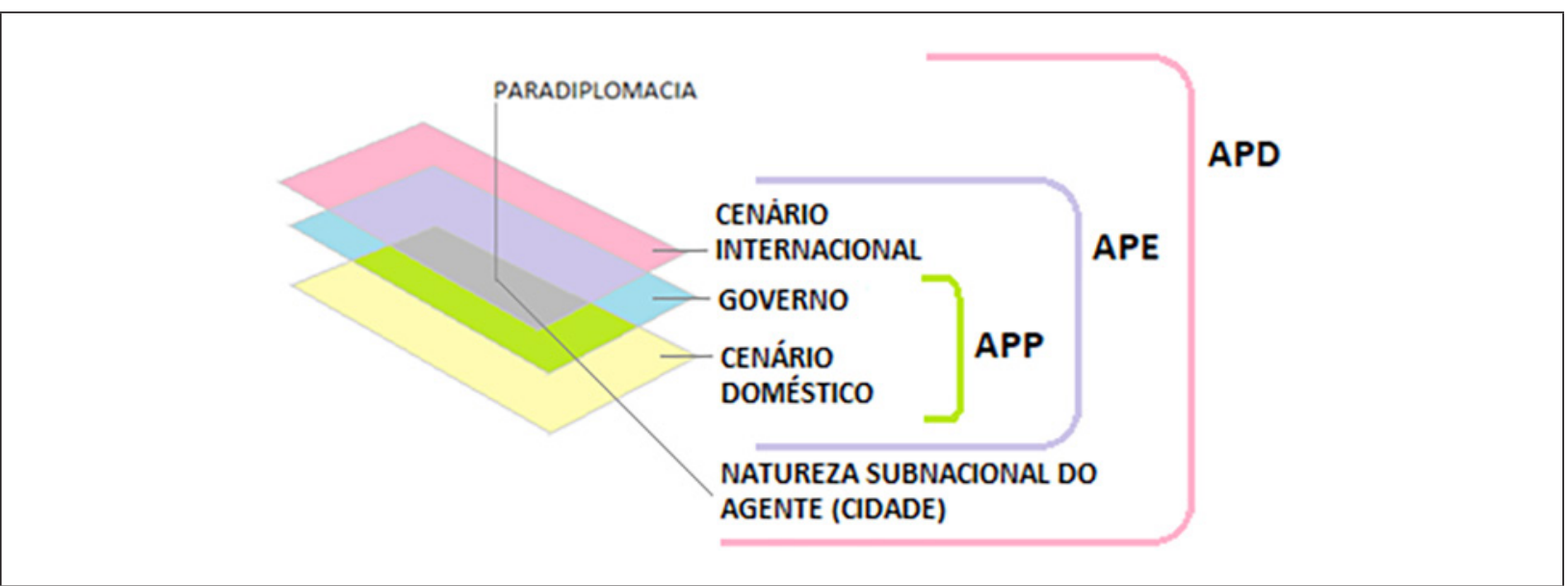

Fonte: elaboração dos autores, 2018.

Da mesma forma que a política externa é considerada como uma política pública (RATTON SÁNCHEZ et al., 2006), a paradiplomacia é considerada política externa (SALOMÓN; NUNES, 2007) e pode ser analisada como política pública. A paradiplomacia possui os mesmos gatilhos e estratégias, como obtenção de ganhos internacionais à população local (SALOMÓN, 2011). Pode-se defender, portanto, a APD a partir do esquema traçado na Figura 1, respeitando as peculiaridades de cada caso empírico analisado.

Talvez uma das mais importantes peculiaridades da APE é seu nível de análise que mescla o doméstico com o internacional - dotados de dinâmicas, responsabilidades, públicos e modalidades de interação de poder distintas. Nesse sentido, a APE e as variáveis a serem analisadas devem sofrer o ajuste de acordo com a natureza do agente a ser estudado. Geralmente, a APE é aplicada para compreender a política externa de Estados. No caso da peculiaridade da natureza das cidades, a realidade subnacional muda de acordo com a nação à qual se inserem, jurídica e politicamente. Além disso, se a análise da paradiplomacia ocorrer em relação a regiões e outros entes federados (que não as cidades), a natureza do agente exige outras adaptações aos modelos de APE e APP.

Estados e cidades possuem diferenças naturais, como nas questões de soberania ou nas limitações jurídicas nacionais. Cidades são livres de soberania (ROSENAU, 1990, p. 36), o que lhes confere maior mobilidade de cooperação nas demais agendas internacionais que não sejam sobre guerra e paz. Por isso, a APE para Estados possui uma distinção em relação à APE aplicada às cidades. Mas nem por isso não existirá competição entre cidades (SÁNCHEZ, 2001) ou hierarquias de recursos de poder, como o financeiro (FRIEDMAN, 1986; SASSEN, 2010). 
Dessa forma, a APD precisa analisar as variáveis da natureza subnacional do agente em questão (seu funcionamento interno, instituições, situação jurídica e política no cenário local e infranacional), as variáveis do cenário doméstico (população local, dinâmicas socioeconômicas, políticas e relação com o governo nacional etc.) e as variáveis do cenário internacional (fluxos financeiros, redes e organizações internacionais, demandas de outras cidades, oportunidades, cooperação e competição por recursos etc.). Assim, ao decidir investigar a paradiplomacia, deve-se ter em mente que os estudos de APE e de APP estão interligados aos estudos de APD e trazem métodos e variáveis explicativas relevantes.

No caso da APP, é importante destacar que ela contribui para a análise sobre os agentes e variáveis relevantes para serem testadas na explicação dos casos de APD. Observar que os gestores locais e grupos de interesses se relacionam com outros agentes formais (como as instituições) e informais (como o mercado) possibilita uma compreensão maior sobre a construção da agenda de políticas públicas e, consequentemente, da política externa e da paradiplomacia. Vale ressaltar que APE e APP não substituiriam as orientações dadas pela literatura especializada sobre paradiplomacia, mas sim complementam a aplicação de métodos e recortes menos usuais nas relações internacionais e fortalecem, por consequência, a APD.

Possivelmente, os primeiros estudos de paradiplomacia que se aproximaram de um olhar mais interno e doméstico nas relações internacionais (aproximando APD à APP) foram os de David Dyment (1993; 2001), que associou a construção dos processos políticos históricos de províncias e cidades canadenses com suas agendas externas desde 1945. Dyment conseguiu, por diversas vezes, demonstrar que não seria possível compreender o local sem olhar para as esferas nacional e global, destacando as simetrias dos componentes culturais na política local e canadense. Suas considerações apontavam para uma compreensão da unidade de análise local como um agente em reflexo às estruturas nacionais e globais. As decisões do governo local seriam explicadas pelas dinâmicas políticas e as de mercado, nos níveis local, regional e internacional.

Do outro lado do Atlântico, nos processos de integração da União Europeia, estudos sobre governos subnacionais e regionalismo despontaram nos anos de 1990 e início dos anos de 2000. Caterina García Segura (2004) analisou os casos da Catalunha, País Basco e Galícia na Espanha. Partindo de uma contextualização histórica, a pesquisadora mostrou como, sob um regime ditatorial e sem democracia, os governos subnacionais são sufocados e não conseguem levar adiante suas 
demandas. No caso, Segura (2004) tentou mostrar como as cláusulas democráticas recentes na Espanha possibilitaram a esses três governos subnacionais recorrerem ao princípio da subsidiariedade e passaram a ter voz sobre os processos que afetam as suas comunidades locais, como a integração regional, altamente determinante na ação externa dos três casos.

Caterina Segura (2004) mostra a importância do reconhecimento dos Estados sobre o diálogo com os governos subnacionais em processos de integração regional, bem como da democracia. Nesse sentido, suas contribuições apontam para se prestar atenção sobre a realidade nacional na qual esses agentes se encontram, bem como para as dinâmicas regionais (internacionais) que podem reforçar e legitimar a ação externa dos governos subnacionais. Assim como David Dyment (1993; 2001), Caterina Segura (2004) reforça o olhar sobre: (i) o contexto sociopolítico nacional; (ii) as relações desses agentes com seus governos nacionais (história política); e (iii) como processos externos ao Estado podem interferir em suas situações locais, como nos casos da integração regional e dos demais fluxos da globalização e interdependência.

Mas essa liberdade, contudo, sob as perspectivas de David Crieckmans (2006), pode levar a outras dinâmicas mais complexas, como no caso de Flandres, na Bélgica. Para o autor, o seu estudo sobre o caso da região de Flandres, no norte da Bélgica, expõe a paradiplomacia como um campo de conflitos de interesses locais e nacionais. Nesse caso, seria possível afirmar que os grupos de pressão internos e as relações socioculturais e políticas que determinam as instituições, as políticas públicas e as políticas externas/paradiplomacias dessas regiões seriam as variáveis com maior capacidade explicativa.

No Brasil, como já mencionado, os estudos de paradiplomacia recorrendo à APE e à APP iniciaram com o estudo de caso das professoras Mónica Salomón e Carmem Nunes (2007). Nessa análise, as autoras diferenciaram a natureza dos agentes "cidade" (Porto Alegre) e "estado federado" (Rio Grande do Sul). Essa diferenciação é importante, visto que, como as autoras apontam, as cidades acabam tendo maior liberdade de atuação por não terem, no Brasil, constituições estaduais que regem questões de segurança e território. As cidades brasileiras também não possuem forças armadas ou policiais. Em suas análises, as professoras concluíram que a alternância partidária no poder de um dos governos subnacionais foi prejudicial para a manutenção da paradiplomacia como política pública, ou seja, da continuidade de agenda e da secretaria especializada em relações internacionais. Por outro lado, a manutenção do mesmo partido político no poder 
manteve a coerência de políticas públicas e política externa por meio da secretaria no outro agente.

Nesse caso, o que se coloca como relevante é a identificação de variáveis partidárias e institucionais - que interferiram na APD. Esse recorte de somas possibilita perceber variáveis que podem interferir nos processos internacionais de uma cidade que parte da literatura de paradiplomacia nas relações internacionais vem ignorando. A pesquisa (SALOMÓN; NUNES, 2007; SALOMÓN, 2012a; SALOMÓN, 2012b) não necessariamente ignora o espaço internacional, mas inicia-se junto ao cenário local e nacional e, posteriormente, abre espaço para a compreensão do externo.

Já os estudos sobre a cidade de São Paulo, como os de Janina Onuki e Amâncio Jorge de Oliveira (2013) ou os liderados por Tullo Vigevani, como a publicação de Nicole Aguilar Gayard (2006/2007), trazem a coleta de dados da Secretaria Municipal de Relações Internacionais de São Paulo (ou inicialmente das assessorias) ao longo de períodos que englobam gestores municipais de partidos distintos, como Marta Suplicy e José Serra. Os estudos relacionam as agendas políticas às práticas da secretaria de relações internacionais de São Paulo. Da mesma forma que Salomón e Nunes (2007), a variável partidária é estabelecida e o cenário político local e nacional. Olha-se um pouco mais para os processos internacionais que alcançam a cidade, colocados como derivados da globalização, e identificam que a própria secretaria gozava de certa autonomia, dado seu corpo técnico-administrativo.

A autonomia institucional das secretarias especializadas contribuiria para a formação de estratégias e agendas internacionais para as cidades. Como Janina Onuki e Amâncio Oliveira (2013) defendem em suas considerações finais:

Esse conjunto de atividades desenvolvidas pela Prefeitura de São Paulo, durante o período 2001-2004, pode ser considerado resultado das ações específicas da Secretaria de Relações Internacionais. Embora parte dessas iniciativas tenha sido gerada pela própria intensificação da interdependência com outros atores internacionais, e pelo incentivo vindo de governos estrangeiros, a estruturação de uma agenda mais sistemática de ação, por parte da Prefeitura, certamente ampliou a capacidade de atender às demandas externas, e estimulou o desenvolvimento de políticas específicas que viria beneficiar diretamente a cidade (ONUKI e OLIVEIRA, 2013, p. 17).

O caso da cidade de São Paulo nesses estudos (ONUKI e OLIVEIRA, 2013; GAYARD; VIGEVANI, 2004 ) apontam para a variável institucional, ou seja, olhar 
para a autonomia das secretarias como fontes de origem e gestão das práticas paradiplomáticas de uma cidade. Contudo, muitos estudos acabam se limitando a olhar apenas para as secretarias, como em análises mais quantitativas - como se isso fosse suficiente. Na próxima seção deste artigo, o objetivo é propor e aplicar um modelo de análise de paradiplomacia capaz de reunir um conjunto de dimensões analíticas que podem ser úteis aos estudos empíricos.

\section{Modelo APD aplicado à Cidade do Rio de Janeiro}

Conforme destacado na introdução, o modelo de Análise de Paradiplomacia (APD) aqui sugerido (Quadro 1) baseou-se na literatura de paradiplomacia, de APE e de APP. Essa literatura aponta para cinco dimensões testadas ou conjuntos de variáveis explicativas, mas fragmentadas em diversos trabalhos: política ou de gestão (SALOMÓN; NUNES, 2007); mercado (SÁNCHEZ, 2001); institucional (SALOMÓN; NUNES; LAISNER, 2007; ONUKI; OLIVEIRA, 2013); dinâmicas e agentes externos ou internacional (SÁNCHEZ, 2001; SASSEN, 2010); e epistêmica (SÁNCHEZ, 2001).

A primeira - aqui denominada política de gestão - trata dos prefeitos, secretários, partidos e outros responsáveis por traçarem ou autorizarem as práticas paradiplomáticas. A segunda dimensão - mercado - analisa variáveis advindas do mercado, do interesse comercial privado, dos fluxos financeiros e das bases econômicas da cidade. A terceira dimensão analisa variáveis institucionais, como o corpo técnico, a autonomia institucional, a especialização e a autoridade diante da APD. A quarta analisa variáveis internacionais, como interferência de organizações, governos nacionais estrangeiros e outros que possam orientar ou determinar as práticas paradiplomáticas. Por fim, a quinta dimensão trata das fontes epistêmicas, como pesquisadores, universidades e grupos de conhecimento (think tanks) que possuem relações diretas com as cidades.

A partir dessas cinco dimensões - gestão política, mercado, institucional, internacional ou agentes externos e epistêmica - , foi possível organizar as variáveis, bem como identificar alguns indicadores mais específicos e algumas técnicas/métodos para coletar os dados. A seguir, explica-se o modelo a ser testado na presente análise de caso sobre a cidade do Rio de Janeiro, onde as cinco dimensões são organizadas em suas variáveis, indicadores e métodos de coleta de dados (Quadro 1). 


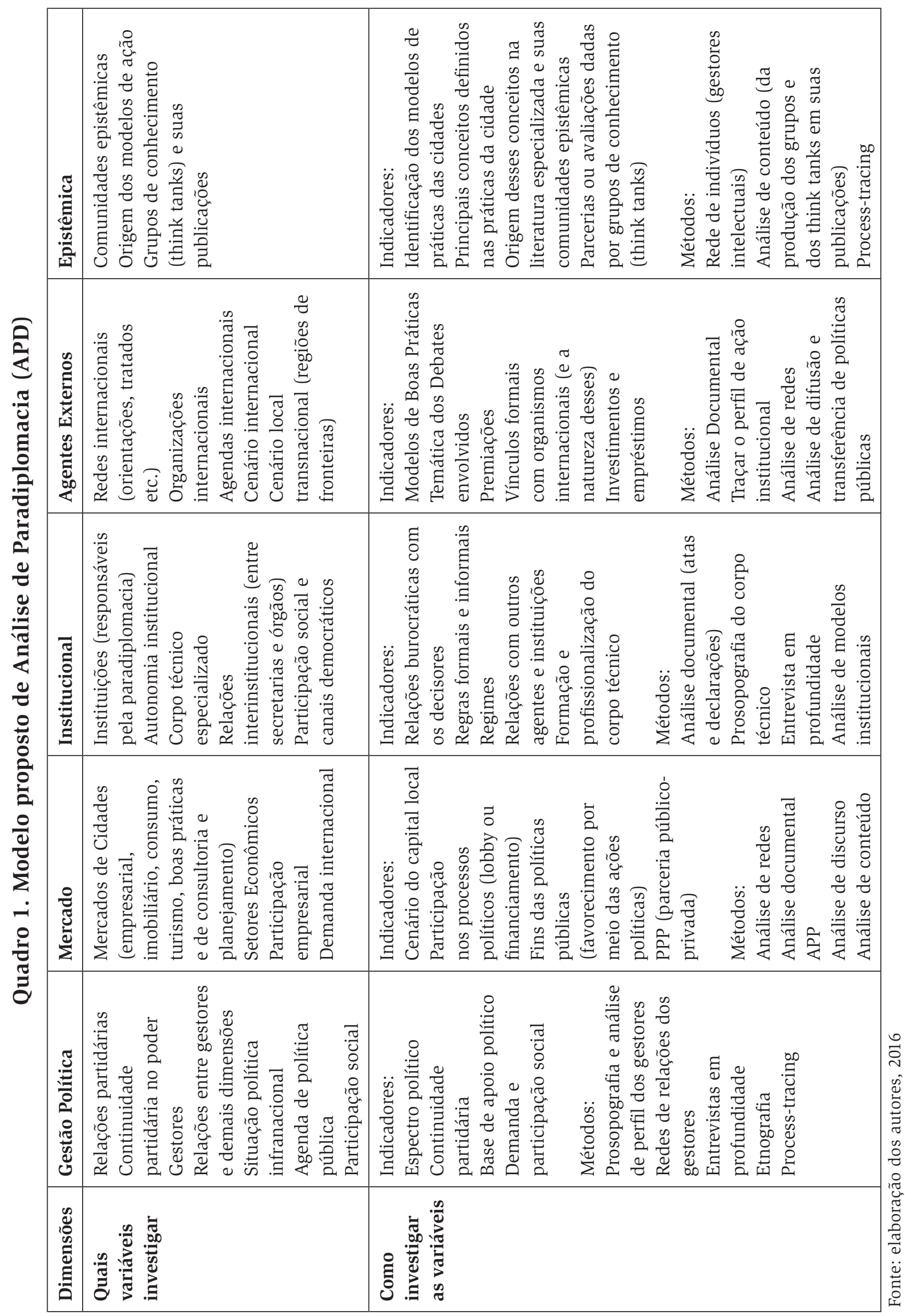


Vale ressaltar que cada dimensão é um conjunto de variáveis que, por sua vez, se sustentam por indicadores que podem variar de acordo com a cidade analisada. Na primeira dimensão, a política de gestão, é investigada a relação entre os gestores e a formulação das políticas públicas (política externa e, consequentemente, a paradiplomacia da cidade), podendo ser utilizada a prosopografia ou estudos de redes, por exemplo, para detectar o envolvimento desses com determinadas temáticas em suas vidas pessoais e entre si. Os estudos partidários podem, também, apontar para rupturas e continuidades. Mas deve-se lembrar que cada variável, em cada caso analisado, pode ter maior ou menor capacidade explicativa da realidade.

Apesar da literatura apontar para descontinuidades paradiplomáticas na medida em que os partidos em oposição se alternam no poder municipal (SALOMÓN; NUNES, 2007; ONUKI; OLIVEIRA, 2013), grupos políticos (elites) podem pertencer a partidos distintos, mas compartilhar os mesmos valores e interesses sobre os resultados de políticas públicas. Nas demais dimensões, como a de mercado, institucional, internacional ou agentes externos e epistêmica, a organização do modelo segue o mesmo padrão.

No caso da dimensão institucional, por exemplo, que possui grande número de pesquisas sobre a interação das instituições nos processos decisórios - como já mencionado - é possível utilizar modelos, como o da escolha racional, o process-tracing, a prosopografia do corpo técnico, entrevistas e análise de relações interinstitucionais para se alcançar os dados. Dessa forma, cada dimensão pode evocar técnicas de suas literaturas especializadas. O objetivo do modelo é indicar possíveis variáveis, indicadores e técnicas adequadas para os estudos empíricos da paradiplomacia.

Na aplicação à cidade do Rio de Janeiro, optou-se pelo recorte de 1993 a 2016. O limite temporal foi estabelecido a partir da implementação do primeiro Plano Estratégico da Cidade que inseriu o campo internacional na agenda municipal de desenvolvimento (1993) até o final do mandado do prefeito Eduardo Paes em 2016. Vale lembrar, mais uma vez, que a escolha da cidade se justifica pela existência de uma continuidade de sua paradiplomacia no período. A explicação dessa continuidade, no entanto, foge do padrão observado nos estudos empíricos que destacaram as variáveis partidárias e institucionais em suas análises (SALOMÓN; NUNES, 2007; LAISNER, 2007; ONUKI; OLIVEIRA, 2013). Ou seja, mesmo verificando-se alternância partidária na prefeitura do Rio, houve a continuidade das práticas da paradiplomacia (MERCHER, 2016). As variáveis partidárias ou institucionais, portanto, não são suficientes para explicar a continuidade no caso 
empírico do Rio. Por isso, diversas variáveis foram testadas para explicar esse caso, usando métodos próprios e conforme o modelo aqui apresentado (Quadro 1).

Propõe-se aqui a APD com cinco dimensões de análise: (I) Gestão Política; (II) Mercado; (III) Institucional; (IV) Internacional; e (V) Epistêmica. Na dimensão de gestão política, as agendas políticas do período foram analisadas, os partidos à frente da Prefeitura do Rio de Janeiro foram identificados e realizou-se uma prosopografia (Quadro 2) dos prefeitos César Maia (1993-1997 e 2001-2009), Luiz Paulo Conde (1997-2001) e Eduardo Paes (2009-2017). Na dimensão de mercado, os agentes empresariais em associações e participações na elaboração das políticas públicas foram identificados, por meio das agendas, órgãos e iniciativas de capital público-privado com a prefeitura. Observou-se, também, a base econômica e as políticas públicas municipais, bem como os conteúdos dos discursos oficiais da Prefeitura do Rio junto aos agentes do mercado.

Na dimensão institucional, as instituições relacionadas à Prefeitura (Executivo e Legislativo) para o campo internacional foram identificadas, bem como analisada a autonomia institucional dessas instituições e o perfil de seus coordenadores. Na dimensão externa, observou-se a participação de agentes internacionais, como organizações do Sistema ONU, e de redes de cidades, como a Mercocidades, por meio de documentos e informativos oficiais. Por fim, na dimensão epistêmica, foram identificados os modelos incorporados na política da cidade, suas origens na produção científica e a relação dos principais grupos de conhecimento envolvidos com a cidade do Rio, como o Centro Brasileiro de Relações Internacionais (CEBRI) e o Grupo Barcelona.

Na Dimensão da Gestão Política (I), investigou-se que partidos ou que gestores (personalismo) teriam maior ou menor capacidade de explicação das estratégias paradiplomáticas. Na variável partidária, uma simples consulta documental aos partidos dos prefeitos e das coligações no legislativo demonstrou que ocorreram mudanças partidárias, bem como coligações de oposições entre os três prefeitos (César Maia, Conde e Paes). Dessa forma, olhando para pesquisas anteriores sobre variáveis partidárias e paradiplomacia (SALOMÓN; NUNES, 2007), essas variáveis deveriam explicar rupturas e não a continuidade vista no caso do Rio (Planos Estratégicos Municipais e as práticas semelhantes ao longo dos governos). Por isso, a variável partidária teve baixa capacidade explicativa. 


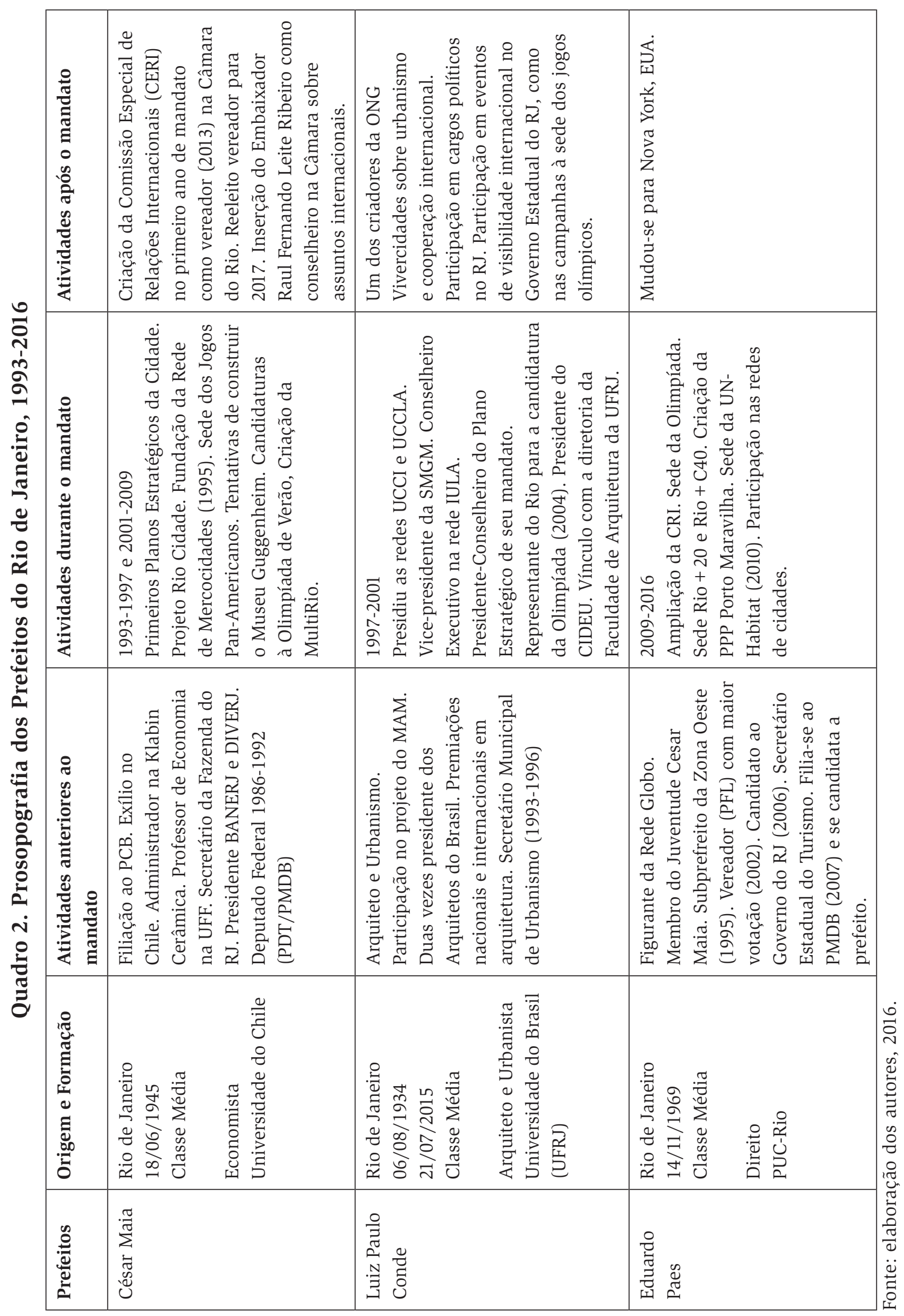

210 Paradiplomacia como Política Externa e Política Pública: modelo de análise aplicado ao caso [...] 
Já a análise dos gestores políticos, por meio da prosopografia (coleta de dados bibliográficos das elites decisórias), demonstrou que, apesar de partidos distintos em planos de governo, manteve-se o mesmo grupo político no poder. A prosopografia dos prefeitos (Quadro 2) indicou, também, o engajamento pessoal com os temas internacionais de cidades, especialmente dos dois primeiros, César Maia e Luiz Paulo Conde em suas atividades anteriores, ao longo e posteriores ao exercício do mandato, como na projeção da estética urbana e celebração de grandes eventos esportivos, artísticos e climáticos à visibilidade internacional. A formação em arquitetura do prefeito Conde reforçou a participação em redes internacionais de urbanismo nos moldes estratégicos já implementados por seu antecessor César Maia: aparelhos arquitetônicos (museus e grandes complexos) atraem visibilidade e investimentos nacionais e internacionais.

Percebe-se que, nessa dinâmica complexa de agentes, internos e externos à cidade do Rio, as variáveis políticas de gestão trouxeram respostas consistentes sobre a ação externa do município. A permanência de um único grupo político no poder, desde 1993 até 2016, revela que os gestores (prefeitos) são os agentes decisores no processo de política externa da cidade. Mas, apesar de determinarem o comportamento da cidade, não criaram os modelos - os absorveram de outros agentes, como do Grupo Barcelona e do CEBRI. Os prefeitos não criam, também, as demandas e grupos de pressões sobre a elaboração de políticas públicas, exigindo uma melhor análise nas demais dimensões do modelo.

Para analisar a Dimensão de Mercado (II), primeiramente identificou-se a base econômica e financeira da cidade, percebendo um predomínio em serviços sobre a indústria. O PIB da capital fluminense é originário da seguinte proporção (IBGE, 2016 ): 65,52\% em serviços; 23,38\% em impostos; 11,06\% em indústria; e $0,3 \%$ em agronegócio. Na pesquisa, foi possível observar que importantes representações comerciais e de interesse privado, como a Associação Comercial do Rio de Janeiro (ACRJ) e a Federação das Indústrias do Rio de Janeiro (FIRJAN), estiveram no Conselho da Cidade e participaram diretamente na definição dos Planos Estratégicos desde 1993, tanto na coordenação como na elaboração dos textos e seus objetivos de desenvolvimento (VAINER, 2001). Os conselheiros também demonstraram vínculos com o mercado de cidades ${ }^{4}$.

4 Fernanda Sánchez (2001) aponta que, no cenário internacional, as cidades competem entre si por recursos e seis interesses econômicos: mercado de empresas localizadas; mercado imobiliário; mercado de consumo; mercado do turismo; mercado das boas práticas; e mercado de consultoria e planejamento. 
Quadro 3. Lista dos Conselheiros da Cidade do Rio de Janeiro, 2016

\begin{tabular}{|c|c|}
\hline O Conselho da Cidade & $\begin{array}{l}\text { "O Conselho da Cidade do Rio de Janeiro é um fórum consultivo para auxiliar a revisão e } \\
\text { o acompanhamento do Plano Estratégico da Prefeitura. Este Conselho é composto por } \\
\text { cidadãos de destacada posição na Sociedade, seja por seu conhecimento das questões } \\
\text { de nosso município, seja por sua contribuição pessoal ou profissional para a evolução de } \\
\text { nossa cidade." Fonte: Conselho da Cidade do Rio de Janeiro, } 2016 \text {. }\end{array}$ \\
\hline Conselheiros & $\begin{array}{l}\text { ALBA ZALUAR - ALBERT ALCOULOUMBRE JR. - ALEXANDRE ACCIOLY - ALEXANDRE } \\
\text { KALACHE - ALEXANDRE SAMPAIO DE ABREU - ALFREDO LOPES - ALFREDO SIRKIS - ALINE } \\
\text { ROMEU XAVIER - ANA PAULA PESSOA - ANA TAVARES DE MIRANDA - ANDRE } \\
\text { EPPINGHAUS - ANGELA MARIA MACHADO DA COSTA - ANGELA ROCHA DOS SANTOS - } \\
\text { ARMANDO STROZENBERG - ASPÁSIA CAMARGO - BERTHA DE BORJA REIS DO VALLE - } \\
\text { BETO SICUPIRA - BORIS LERNER - BRENDA VALANSI OSÓRIO - BRUNO FILIPPO - BRUNO } \\
\text { TYSCHLER - CACÁ DIEGUES - CARLA CAMURATI - CARLA PINHEIRO CARLOS ARTHUR } \\
\text { NUZMAN - CARLOS CARVALHO - CARLOS EDEN SARDENBERG MESQUITA - CARLOS } \\
\text { TUFVESSON - CARMEN FONTENELLE - CAROL SAMPAIO - CAROLINA WAMBIER - CELINA } \\
\text { CARPI - CELSO BARROS - CESAR CUNHA CAMPOS - CEZAR ROGERIO VASQUEZ - } \\
\text { CHRISTIANE PAQUELET - CHRISTINA BARBOSA - DANIEL OELSNER - DANIELA FISZPAN - } \\
\text { DAVID CARDEMAN - DAVID ZYLBERSZTAJN - DELAIR DUMBROSCK - DOM JOÄO ORANI } \\
\text { TEMPESTA - EDGAR FLEXA RIBEIRO - EDMUNDO MUSA - EDSON DINIZ NÓBREGA JUNIOR } \\
\text { - EDUARDA LA ROCQUE - EDUARDO FERREIRA REBUZZI ELENA LANDAU - ELOI } \\
\text { FERNÁNDEZ Y FERNÁNDEZ - ERNANI DE SOUZA COSTA - FÁBIO PALMA - FELIPE SANTA } \\
\text { CRUZ - FERNANDA MONTENEGRO - FERNANDO MACDOWELL [ET aI] }\end{array}$ \\
\hline $\begin{array}{c}\text { Informações dos } \\
\text { Conselheiros }\end{array}$ & $\begin{array}{l}\text { Ângela Maria Machado da Costa (Presidente do Conselho Empresarial de Gestão } \\
\text { Estratégica para Competitividade, ela que, em 2005, foi eleita pela Revista Forbes } \\
\text { como "A Mulher Mais Influente do Brasil" na categoria Indústria e Varejo); Carlos } \\
\text { Alberto Veiga Sicupira (considerado o quarto homem mais rico do Brasil em } \\
2016 \text {, sócio da companhia belga-brasileira Anheuser-Busch Inbev. Essa empresa } \\
\text { é a maior cervejaria do mundo, dona de } 25 \% \text { do mercado global, além de ser uma } \\
\text { das cinco maiores empresas do mundo de produtos de consumo. Ele também é } \\
\text { associado à } 3 G \text { Capital, Warren Buffett, Burger King e Heinz); Celina Carpi } \\
\text { (membro do Conselho de Administração da Libra Holding, operadora portuária e } \\
\text { de logistica de comércio exterior e participa da diretoria da FIRJAN); Elena } \\
\text { Landau (economista neoliberal que foi assistente da presidência do BNDES); } \\
\text { Gabriel Bello Barros (responsável pela cooperação bilateral da CRI); Gilbert } \\
\text { D’Orey Landsberg (Sênior Eternal Affairs da Shell); Guilherme Rodolfo Laager } \\
\text { (Diretor de Logistics and Director of IT, Cervejarias Kaiser Brasil S/A do Grupo } \\
\text { Heineken); Hugo Barreto (Secretário Geral da Fundação Roberto Marinho); João } \\
\text { Dionísio Amoêdo (Criador do Partido Novo em espectro liberal); João Paulo Rio } \\
\text { Tinto de Matos (presidente eleito da Associação dos Dirigentes do Mercado } \\
\text { Imobiliário do Rio); José Formoso (ex-presidente da Embratel); José Luiz } \\
\text { Alqueres (Grande Benemérito e presidente da Associação Comercial do Rio de } \\
\text { Janeiro, ACRJ, ex-presidente do Conselho de Administração da Eletrobrás); } \\
\text { Laudemar Aguiar Neto (Diplomata e Coordenador da CRI); Roberta Medina } \\
\text { (empresária e produtora de eventos, filha do criador do Rock in Rio, Roberto } \\
\text { Medina); Tomas Tomislav|Antonin Zinner (presidente do Unibanco até 1998, } \\
\text { atualmente conselheiro Unibanco/Itaú) [et al] }\end{array}$ \\
\hline
\end{tabular}

Fonte: elaboração dos autores, 2016. 
Os documentos do Conselho da Cidade demonstraram que 47 de seus 217 conselheiros pertenciam às principais empresas vinculadas aos mercados de cidades, como Roberta Medina e Carlos Alberto Veiga Sicupira (Quadro 3). Isso representa uma porcentagem de aproximadamente $22 \%$ dos conselheiros. Mas essa porcentagem não engloba todos, visto que, além desses grandes empresários, existem, ainda, os advogados sócios em firmas de advocacia e empresários de médio e pequeno porte, como designers e lojistas.

Os grandes empresários formam a maior e mais homogênea parte do grupo do Conselho da Cidade, representando grandes empresas nacionais e internacionais. Esse grupo é seguido pelos urbanistas, economistas, advogados e pequenos e médios empresários. Depois desses estão acadêmicos, esportistas, profissionais da saúde, artistas e políticos - as "personalidades da Cidade”. Os dados do Conselho da Cidade materializam de forma clara a relação entre o mercado e os Planos Estratégicos da Cidade, ainda mais que a FIRJAN e ACRJ. Essas duas instituições, além de redigir e coordenar o processo de criação dos Planos Estratégicos do Rio no Conselho da Cidade, estariam duplamente representadas, uma vez que muitos de seus membros estão ali como conselheiros. Não por acaso, as práticas de sediar grandes eventos que atraiam turistas, desenvolvam o comércio e aqueçam o mercado imobiliário sempre se mantiveram nos objetivos e práticas paradiplomáticas da cidade do Rio de Janeiro no período analisado.

Contudo, não foi o mercado quem criou o modelo. No máximo, financiou, demandou ou apoiou a sua criação e implementação pelos gestores políticos. Por isso, a análise das dimensões Institucional (III), Internacional (IV) e Epistêmica (V) foram realizadas. Na Institucional, os órgãos municipais responsáveis pela paradiplomacia (Coordenadoria de Relações Internacionais da Cidade do Rio de Janeiro - CRI e a Comissão Especial de Relações Internacionais da Câmara dos Vereadores do Rio de Janeiro - CERI) e suas práticas foram investigadas por meio de análise documental e da prosopografia de seus dirigentes (Quadro 4). 


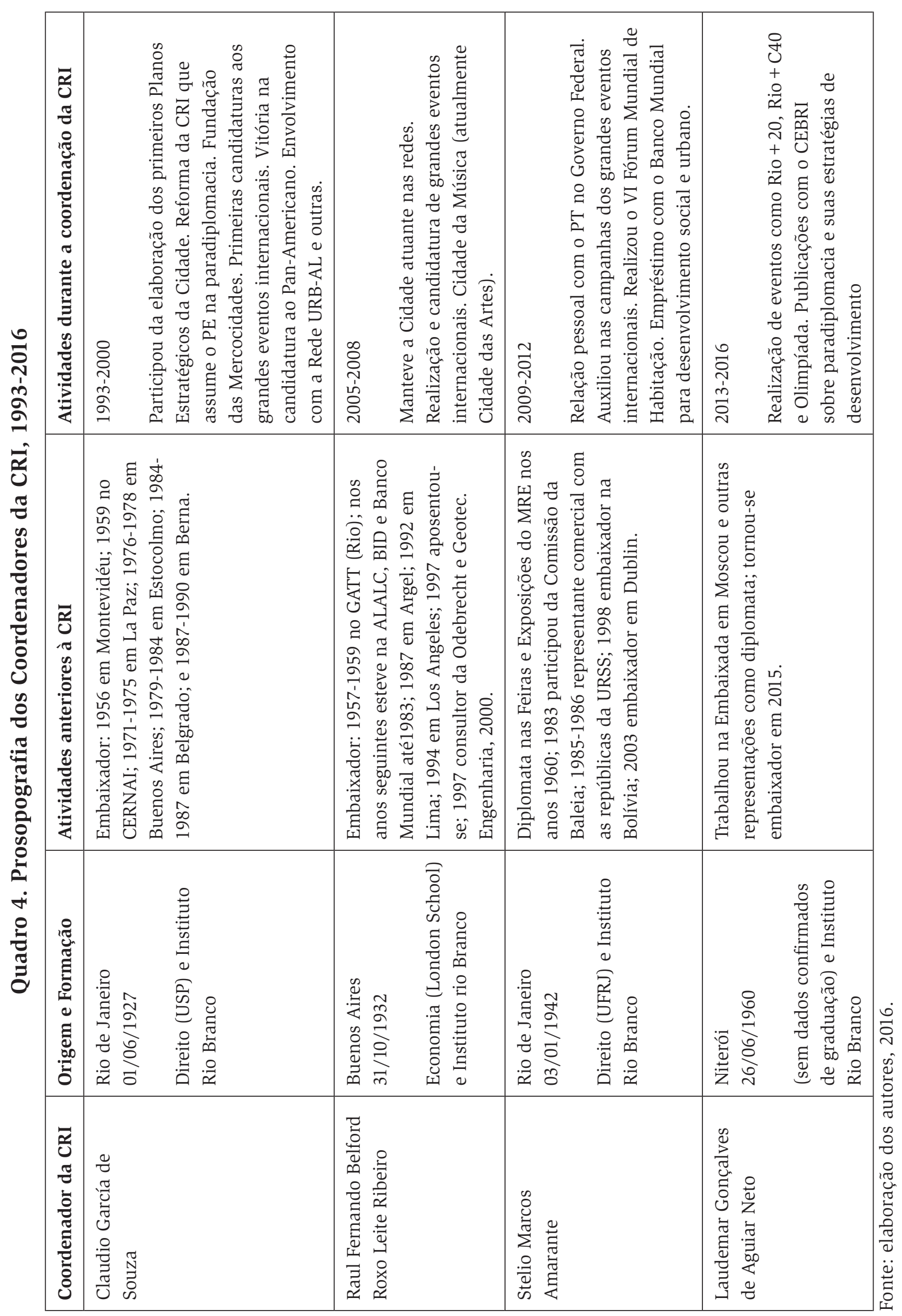


Os resultados da análise Institucional (III) apontaram para alta capacidade técnica (funcional), mas baixa autonomia em relação aos gestores (prefeitos). Por exemplo, os cargos de chefia da Coordenadoria de Relações Internacionais da Cidade do Rio de Janeiro (CRI) sempre foram ocupados por diplomatas de carreira no período analisado, mas com alternâncias de acordo com os mandatos dos prefeitos. Com exceção do embaixador Claudio Garcia de Souza, o cargo de coordenador da CRI sempre teve rotatividade para cada novo mandato de prefeito. É possível observar, também, baixa autonomia institucional por causa do vínculo da CRI com o Gabinete do Prefeito no Palácio da Cidade (orçamento, indicações, agenda direta do prefeito etc.). Portanto, a CRI se coloca como o principal órgão de convênios e ações paradiplomáticas da cidade, mas se submete diretamente ao prefeito e aos Planos Estratégicos da Cidade - que advêm do Conselho da Cidade (mercado e sociedade).

Já no Poder Legislativo municipal as tentativas de César Maia de criar uma Comissão de Relações Internacionais nos últimos anos não demonstraram fôlego para orientar as práticas paradiplomáticas da Cidade, mantendo, ainda, a centralização paradiplomática na CRI (Gabinete do Prefeito). Mas a criação da Comissão Especial da Câmara dos Vereadores por César Maia (primeiro ato após eleito como vereador depois de concluir seu mandato na prefeitura) ilustra que a agenda internacional da cidade em seus mandatos à frente do Executivo não era apenas um tema secundário, mas de atenção pessoal, demonstrada nos seus discursos e posicionamentos sobre as relações internacionais do Rio.

Sobre a dimensão dos agentes externos e interferências internacionais (IV), observou-se demanda de redes de cidades (Mercocidades, CGLU etc.) e organismos internacionais em cooperar e firmar parcerias com o Rio de Janeiro, como no empréstimo adquirido, em 2009, do Banco Mundial. Identificar os agentes e as dinâmicas foi o primeiro passo, seguido da análise documental e de seu cruzamento com apontamentos teóricos da literatura especializada. Mas, de todas as dimensões, essa demonstrou ser a mais dependente dos interesses pré-definidos na agenda internacional da cidade pela Prefeitura. Empréstimos e participações do Rio no âmbito internacional só ocorreram na medida em que os gestores entendiam ou percebiam vantagens.

A autonomia de decisão em relação aos agentes internacionais se mostrou mais elevada do que, por exemplo, diante dos grupos de pressão comerciais locais. Entretanto, sem as variáveis internacionais e agentes externos, pouco se 
compreenderia sobre o fortalecimento da estratégia paradiplomática adotada. Por exemplo, a busca por sediar grandes eventos e conseguir empréstimos e financiamentos de organizações internacionais pode ser compreendida como mecanismos estratégicos de competição internacional em relação a outras cidades. Mas as cidades competem se os gestores (e seus grupos de pressão) perceberem vantagens e assim decidirem. Dessa forma, os mercados de cidades apontados por Fernanda Sánchez (2001), como o mercado de boas práticas ${ }^{5}$, e as dinâmicas dos fluxos de capitais na globalização, identificados por Saskia Sassen (2010), ajudam a entender que o cenário internacional se coloca como uma variável interveniente, que induz a concorrência entre governos locais, mas não necessariamente determinaria as ações do Rio - e, possivelmente, as ações de muitas outras cidades.

Referente aos resultados da análise da Dimensão Epistêmica (V), observou-se o conteúdo dos Planos Estratégicos e das ações da cidade para identificar ideologias, valores e ideias compartilhadas pelos gestores e grupos sociais de pressão. Pesquisadores como Fernanda Sánchez (2001) e Claudio Vainer (2001) já haviam mencionado que os modelos de desenvolvimento municipal do Rio e, consequentemente, a sua paradiplomacia, vieram de experiências anteriores e de boas práticas da cidade de Barcelona, intermediadas pelo político e professor catalão Jordi Borja (TUBSA S.A. ou Grupo Barcelona). Desde 1993, Borja inseriu o modelo que deu origem aos Planos Estratégicos e ao conselho da cidade, tal qual se desenvolveu nesses 23 anos, como, por exemplo, tendo as representações comerciais - ACRJ e FIRJAN - espaço de coordenação e liderança (VAINER, 2001).

Além disso, durante as investigações institucionais, foi possível perceber que grupos de conhecimento (think tanks), como o Centro Brasileiro de Relações Internacionais (CEBRI), demonstraram vínculo institucional direto com a CRI, onde suas publicações apontam modelos de paradiplomacia, bem como existem publicações do próprio corpo técnico da Prefeitura em relações internacionais. O artigo do Coordenador da CRI, o Diplomata Laudemar Aguiar (2013), durante o governo de Eduardo Paes, reafirmava as ideias estratégicas de desenvolvimento

5 Segundo Fernanda Sánchez (2001), o Banco Mundial premia boas práticas em políticas públicas, o que aquece o mercado da consultoria e impulsiona, também, outras cidades a pegarem empréstimos para aplicarem nos modelos premiados pelo Banco Mundial - muitas vezes, o mesmo que oferta o empréstimo. Essa situação pode criar um ciclo de conceitos de desenvolvimento, comércio de modelos e endividamento das cidades. 
dos Planos Estratégicos, do Grupo Barcelona e de outros pesquisadores no CEBRI. Além disso, pesquisadores em contraponto (críticos ou opositores intelectuais) aos modelos adotados pela cidade nesse período, como Carlos Vainer (UFRJ) e Fernanda Sánchez (UFF), tiveram pouca repercussão na gestão estratégica e paradiplomática do Rio de Janeiro.

Nas concepções críticas da academia local, as dimensões de gestão política, mercado e institucional demonstraram o alinhamento com uma vertente conceitual neoliberal (SÁNCHEZ, 2001; VAINER, 2001), que compreende a paradiplomacia como ferramenta de captação de recursos e desenvolvimento financeiro e comercial de uma cidade. A reforma urbana e o investimento em aparelhos arquitetônicos e grandes eventos que deram visibilidade internacional trariam investimentos de capital que, posteriormente, poderiam ser convertidos em financiamento de políticas sociais ou ambientais. Essa perspectiva, contudo, não está abertamente presente no conteúdo das publicações do CEBRI. Mas, o conteúdo do CEBRI (na forma de artigos publicados) reproduz o da CRI e vice-versa sobre paradiplomacia como ferramenta do desenvolvimento por meio da visibilidade e da atração de capital internacional. Não é de se estranhar que as relações entre CRI (Prefeitura do Rio) e o CEBRI vão além de publicações, onde o próprio CEBRI reconhecia (até 2016) a Prefeitura do Rio como uma de suas mantenedoras institucionais, juntamente com outras empresas - presentes indiretamente no Conselho da Cidade.

De fato, a existência de uma instituição epistêmica na cidade (CEBRI) contribui para a organização e continuidade da ação paradiplomática da forma que foi implementada desde 1993 pelo Grupo Barcelona. Mas não necessariamente determina sua implementação pelos gestores. A escolha dos gestores no Rio (prefeitos e coordenadores da CRI) de adotarem essas ideias de grupos de conhecimento poderia ser explicada pelas variáveis já apresentadas anteriormente: formação profissional dos gestores; e grupos de pressão sobre a elaboração de políticas públicas. Assim, no caso do Rio - e talvez de muitas outras cidades -, as variáveis determinantes da estratégia paradiplomática sejam as vinculadas aos gestores e aos agentes econômicos presentes em suas regiões. Por isso, os estudos de APP e APE podem facilitar as investigações em APD. 


\section{Considerações finais}

Com o intuito de facilitar a compreensão do processo de pesquisa em paradiplomacia, apresentou-se aqui o modelo de Análise de Paradiplomacia (APD) que reúne elementos de Análise de Políticas Públicas (APP) e Análise de Política Externa (APE). Baseado no modelo de APE de Mónica Salomón e Letícia Pinheiro (2013) e nos modelos de APP de Celina Souza (2006) e Enrique Saraiva (2007), cinco dimensões de análise foram reunidas e destacadas como as variáveis mais relevantes apontadas pela literatura especializada, bem como foram apresentados alguns métodos de coleta de dados. Para ilustrar sua aplicação, o caso da cidade do Rio de Janeiro foi apresentado, onde as dimensões política de gestão e de mercado tiveram grande poder explicativo.

Para cada uma das cinco dimensões propostas - gestão política, mercado, institucional, internacional e epistêmica - diversas variáveis foram testadas, apontando para resultados diferentes de outros trabalhos já apresentados na disciplina. Enquanto que a variável partidária e institucional conseguiu explicar casos de continuidade estratégica de cidades brasileiras (SALOMÓN; NUNES, 2007; LAISNER, 2007; ONUKI; OLIVEIRA, 2013), o caso do Rio demonstrou que variáveis como profissionalização e carreira dos gestores, grupo político e agentes comerciais conseguem explicar a continuidade observada na prática paradiplomática do Rio de Janeiro durante o período de 1993 a 2016.

Nesse sentido, o modelo apresentado (Quadro 1) busca reunir e flexibilizar as variáveis e os métodos de coleta e análise de dados tendo em mente que as cidades, apesar de terem em comum a natureza governamental, possuem peculiaridades próprias que desafiam perspectivas mais limitadas em uma ou duas dimensões. Seguindo as premissas de que a APE e a APP são mais do que métodos - mas um conjunto de valores sobre como compreender a realidade de forma plural e com diversos níveis de análise - , foi elaborada a APD que incentiva a análise somada dos cenários doméstico, internacional e da natureza do agente diante das relações internacionais.

A relação dos estudos de APE e APP com os de APD, portanto, exige um olhar para dentro das cidades em suas diversas dimensões. A concepção adotada, de que a paradiplomacia é em si política externa e que esta, por sua vez, é uma política pública, ampliou o número de variáveis a serem investigadas. Dos gestores aos empresários e à demanda dos mercados, passando pela autonomia institucional, 
pelo corpo técnico e pela interferência de agentes nacionais e internacionais, as variáveis foram organizadas no modelo em suas cinco dimensões, mas que não devem ser vistas como rígidas. Dessa forma, a APD e a APP, assim como já ocorre com a APE, devem ser vistas como recursos analíticos relevantes para a compreensão da multiplicidade de agentes e suas formas de participação nas relações internacionais contemporâneas.

\section{Referências}

AGUIAR, Laudemar; MENDES, Anna Carolina; SPADALE, Pedro. A inserção internacional do Rio de Janeiro. CEBRI Artigos, Ed. Especial, v. 3, 2013, p. 1-36. Disponível em: < http://www.cebri.org/portal/publicacoes/cebri-artigos/a-insercao-internacionaldo-rio-de-janeiro > . Acesso em: 15 de abr. 2018.

ALliSON, Graham. Conceptual Models and the Cuban Missile Crisis. The American Political Science Review, v. 63, set. 1969, p. 689-718.

CGLU, Cidades e Governos Locais Unidos. Level of decentralization by country, 2016. Disponível em: < https://www.uclg.org/sites/default/files/plantilla_regiones_en.pdf > . Acesso em 15 de abr. 2018.

CRIEKEMANS, David. How subnational entities try to develop their own 'paradiplomacy'. The case of Flanders (1993-2005). In: International Conference Challenges for Foreign Ministries: Managing Diplomatic Networks and Optimising Value. Geneva, maio/ jun. 2006. Disponível em: < paradiplomacia.org/upload/downloads/418b10efdd1b 499d20e71ddee72a698bparadiplomacy.pdf > . Acesso em: 24 de set. 2018.

DUCHACEK, Ivo. Perforated Sovereignties: Towards a Typology of New Actors in International Relations. In: MICHELMANN, Hans e SOLDATOS, Panayotis. (Org.) Federalism and International Relations: the role of subnational units. Oxford: Claredon Press, 1990, p. 01-33.

DYMENT, David. Substate Paradiplomacy: the case of the Ontario Government. In: HOCKING, Brian (Org.) Foreign Relations and Federal States. Londres: Leicester University Press, 1993.

DYMENT, David. The Ontario Government as an International Actor. Londres: Regional \& Federal Studies, v. 11, n. 1, 2001, p. 55-79.

FEARON, James. Domestic politics, foreign policy, and theories of international relations. Annual Review of Political Science, 1998, p. 289-313.

FRIEDMAN, John. The World City Hypothesis. Londres: Development and Change, v. 17 , jan. 1986, p. 69-83. 
GAYARD, Nicole Aguilar; VIGEVANI, Tullo. A paradiplomacia na Cidade de São Paulo: uma análise da atuação do município no contexto internacional. In: II Relatório Científico FAPESP: Gestão Pública e inserção internacional das cidades. São Paulo: CEDEC/ PUC-SP/ UNESP, 2007.

HOCKING, Brian. Localizing Foreign Policy: Non-central Governments and Multilayered Diplomacy. Londres e Nova York: Macmillan and Saint Martin’s Press, 1993.

HUDSON, Valerie; VORE, Christopher. Foreign Policy Analysis Yesterday, Today, and Tomorrow. Mershon International Studies Review, v. 39, out. 1995, p. 209-238.

IBGE, Instituto Brasileiro de Geografia e Estatística. Cidades: Rio de Janeiro, 2016. Disponível em < https://cidades.ibge.gov.br/ > . Acesso em: 24 de set. 2018.

LAISNER, Regina. A democracia em rede: a URB-AL e os processos de cooperação internacional para a implantação da democracia participativa local. In: VIII Encontro Internacional do Fórum Universitário do Mercosul: Desafios e oportunidades da integração regional no norte da América do Sul, Universidade Federal de Sergipe (UFS), Aracaju, 2007.

MERCHER, Leonardo. Paradiplomacia do Rio de Janeiro: variáveis explicativas à política externa de uma cidade. Tese de Doutorado apresentada no Programa de PósGraduação em Ciência Política da Universidade Federal do Paraná em 16 de dez. 2016. Disponível em: < http://www.cienciapolitica.ufpr.br/ppgcp/wp-content/uploads/ sites/4/2017/02/Tese-Leonardo-M\% C3 \% A8rcher.pdf > . Acesso em: 15 de abr. 2018.

MILNER, Helen; KEOHANE, Robert. Internationalization and Domestic Politics. Cambridge: Cambridge University Press, 1996.

MILNER, Helen. Interests, Institutions and Information: Domestic Politics and International Relations. Princeton: Princeton University Press, 1997.

ONUKI, Janaina; OLIVEIRA, Amâncio Jorge. Paradiplomacia e Relações Internacionais: a experiência da cidade de São Paulo. São Paulo: Centro de Estudos das Negociações Internacionais, USP, Série Working Paper, maio de 2013.

RATTON SANCHEZ, Michelle; SILVA, Elaine; CARDOSO, Evorah; SPECIE, Priscila. Política externa como política pública: uma análise pela regulamentação constitucional brasileira (1967-1988). Curitiba: Revista Sociologia Política, n. 27, nov. 2006, p. 125-143.

RIBEIRO, Maria Clotilde Meireles. Globalização e novos atores: a paradiplomacia das cidades brasileiras. Salvador: EDUFBA, 2009, p. 69-136.

ROSENAU, James. The premises and promises of decision-making analysis. Nova York: Contemporary Political Analysis, Free Press, 1967, p. 189-211.

ROSENAU, James. Turbulence in world politics. Nova Jersey: Princeton University Press, 1990.

SALOMÓN, Mónica. Teorias e enfoques das Relações Internacionais: uma introdução. Curitiba: Intersaberes, 2016. 
SALOMÓN. Mónica. Cooperação sul-sul e sul-norte de cidades. In: Seminário Internacional Agendas e Atores: perspectivas do Norte e do Sul, Rio de Janeiro, Instituto de Relações Internacionais, PUC-Rio, 08-09 de set. 2011. Disponível em: < https://www.youtube. com/watch?v = hlMZjYOIx8I > . Acesso em: 25 de abr. 2018.

SALOMÓN, Mónica. A dimensão subnacional da política externa brasileira: determinantes, conteúdos e perspectivas. In: PINHEIRO, Leticia; MILANI, Carlos (Org.). Política Externa Brasileira: a prática das políticas e a política das práticas. Rio de Janeiro: Fundação Getúlio Vargas, 2012a, p. 269-300.

SALOMÓN, Mónica. Em que medida é possível integrar a Cooperação Descentralizada na dimensão Sul-Sul da política externa brasileira? Rio de Janeiro: Mural Internacional, v. 3, n. 2, 2012b, p. 9-15.

SALOMON, Mónica; NUNES, Carmen. A Ação Externa dos Governos Subnacionais no Brasil: Os Casos do Rio Grande do Sul e de Porto Alegre. Um Estudo Comparativo de Dois Tipos de Atores Mistos. Rio de Janeiro: Contexto Internacional, v. 29, n. 1, 2007, p. 99-147.

SALOMÓN. Mónica; PINHEIRO, Letícia. Análise de política externa e política externa brasileira: trajetória, desafios e possibilidades de um campo de estudos. Brasília: Revista Brasileira de Política Internacional, v. 56, 2013, p. 40-59.

SÁNCHEZ, Fernanda. A Reinvenção das cidades na virada do século: agentes, estratégias e escalas de ação política. Curitiba: Revista de Sociologia e Política, ed. Cidade e Poder, n. 16, jun. 2001, p. 31-49.

SARAIVA, Enrique. O Conceito de Política Pública. In: SARAIVA, Enrique; FERRAREZI, Elisabete (Org.) Políticas Públicas: Coletânea, Brasília, Escola Nacional de Administração Pública, 2007, p. 19-42.

SASSEN, Saskia. Sociologia da globalização. Porto Alegre: Artmed, 2010.

SEGURA, Caterína García. La participación de las comunidades autónomas españolas en las relaciones internacionales. Reflexiones sobre la presencia internacional de las comunidades autónomas históricas: Cataluña, Galicia y el País Vasco. In. VIGEVANI, Tullo; WANDERLEY, Luiz Eduardo; BARRETO, Maria; MARIANO, Marcelo (Org.) A dimensão subnacional e as Relações Internacionais. São Paulo: Educ/Unesp/ EdUSC/FAPESP, 2004.

SOLDATOS, Panayotis. An Explanatory Framework for the study of Federated States as Foreign Policy Actors. In: MICHELMANN, Hans; SOLDATOS, Panayotis (Org.) Federalism and International Relations: The role of subnational Units. Oxford: Clarendon Press, 1990, p. 34-53.

SOUZA, Celina. Políticas Públicas: uma revisão da literatura. Sociologias, Porto Alegre, n. 16, dez. 2006. 
VAINER, Carlos. Os liberais também fazem planejamento urbano? Glosas ao "Plano Estratégico da Cidade do Rio de Janeiro". In: ARANTES, Otília; VAINER, Carlos B.; MARICATO, Ermínia (Org.) A cidade do pensamento único; desmanchando consensos. Petrópolis: Vozes, 2001, p. 105-120.

VIGEVANI, Tullo. El marco jurídico e institucional para la gestión internacional de los actores subnacionales gubernamentales em Brasil. Integración \& Comércio, n. 21, 2004, p. 27-46.

WALTZ, Kenneth. Theory of International Politics. New York: McGraw Hill, 1979.

WENDT, Alexander. Anarchy is what States make of it: the social construction of power politics. International Organization, 46 (spring), 1992, p. 391-425.

WHITE, Brian. The European Challenge to Foreign Policy Analysis. European Journal of International Relations, 1999, p. 35-66. 